\title{
Estimating the effect of early discharge policy on readmission rate. An instrumental variable approach
}

\section{Eugenia Amporfu}

Kwame Nkrumah University of Science and Technology, Kumasi, Ghana; eamporfu@gmail.com

Received 23 January 2010; revised 7 February 2010; accepted 8 February 2010.

\begin{abstract}
*
Early discharge policy, common in the developed countries, refers to the reduction of hospital length of stay as a way of reducing the cost of care. The effect of the policy on quality of care has received a lot of attention in the literature. Some of the earlier papers have ignored the endogeneity of length of stay in the readmission equation, an approach that could lead to inconsistent estimation. This study develops a statistical technique for the consistent estimation of the effect of the early discharge policy. An instrument that can be used extensively across different diagnostic groups is provided, hence solving the difficult problem of finding an instrument for length of stay. The exogeneity test in Gorgger (1990), the test for weak instruments in Staiger and Stock (1997) as well as the Hensen (1982) for over identification confirmed respectively that length of stay is endogenous the instrument is strong and the valid.
\end{abstract}

Keywords: Instrument; Length of Stay;

Early Discharge; Endogeneity;

Instrumental Variable Estimation

\section{INTRODUCTION}

Instrumental variable estimation is a two stage estimation technique that first purges endogenous regressors, and hence exogenizes them before including them as regressors in a regression equation for estimation. An important reason why instrumental variable estimation is becoming less popular is the difficulty of finding an instrument. This difficulty is encountered in models that measure the impact of early discharge policy on quality of care. Many studies have thus ignored the need for instrumental variable estimation and hence the consis-

*Data used for this study was provided by British Columbia (Canada) Ministry of Health and Center for Health Services and Policy Research linked database. tency of their estimated impact of the policy is questionable. The objective of this paper is to develop a statistical technique for consistent estimation of the impact of early discharge on quality of care regardless of the diagnostic group used. The British Columbia (Canada) Ministry of Health and Center for Health Services and Policy Research linked database provided the maternity data used for the study.

The models that measured the impact of the early discharge policy on quality of care used readmission and mortality rates as proxies for quality of care with length of stay as one of the independent variables. Length of stay, because it is correlated with an unobservable variable, the severity of illness, is endogenous in the regression equation. Severity of illness is unobservable to the researcher but observable to the patient (or doctor) and a severely ill patient is likely to stay long in the hospital and be readmitted. Ignoring the endogeneity of length of stay then can lead to inconsistent estimation and hence unreliable conclusions. Examples of papers that ignored the endogeneity of length of stay in the readmission equation can be cited [1].

Consistent estimation of the impact of early discharge policy on quality of care is important because the policy is implemented extensively across North America and Europe (e.g., Sweden, Norway in the 1990s), as a cost containment strategy. In the United States the policy was induced by the introduction of prospective payment [2] and capitation [3] in the 1980s. In Canada, the policy was introduced by provincial governments in the 1990s (example, 1994 in British Columbia and Alberta) and was often accompanied by home visits implemented through public health nursing programs. In general, the early discharge policy has raised concern about its impact on quality of care and has attracted a lot of studies in the area $[2,4]$.

Information on the impact of the policy on quality of care will serve as an important guide to policy makers on the extent to which the policy is able to reduce the cost of care. Inconsistent estimation of the impact then can lead to misleading results and hence mislead policy makers on the efficiency of the allocation of health care 
resources. The endogeneity problem can be solved if severity of illness can be accurately measured and included in the regression equation. This requires clinical information from medical records [5]. Such an approach is however not used in the literature because it is extremely costly to gather the information [6].

Some studies such as [7] used the cost weight of patients diagnostic group, hospital entry type (i.e., whether it was emergency or elective) and whether the diagnostic group was a surgical or non surgical type as proxies for severity of illness. These variables are reasonable proxies for the study's data: all elderly patients across different diagnostic groups. Such variables however cannot pass as proxies for all diagnostic groups especially maternity data. This is because for maternity, an emergency entry usually implies advancement in labor but does not necessarily imply severity of illness. Caesarean section could occur by choice, or as a result of the woman's pelvic or birth canal or for medical reasons. Thus a woman who undergoes caesarean section is not necessarily ill. Hence surgical delivery cannot be an indicator of severity of illness for a maternity patient and the difficulty of finding a proxy for the severity of illness remains. Finding an appropriate instrument for length of stay then would lead to a consistent estimation of the policy's impact on quality of care and nullify the need to find a proxy for severity of illness.

The first to alert of the endogeneity problem with length of stay and the need to use instrumental variables to ensure consistent estimation did not come till 2000 [8]. However, [8] used time of delivery and the method of delivery as instruments for length of stay. While these instruments might have produced consistent estimates, the instruments chosen are only restricted to the data set used, newborns, and so cannot be applied to other diagnostic groups. It is not surprising that more recent papers [1] that did not use infant data ignored the endogeneity problem. What is needed then is an instrument that is highly correlated with length of stay but uncorrelated with severity of illness regardless of the diagnostic group used for the analysis of effect of the early discharge policy. Such an instrument is very important because the early discharge policy is widely implemented for different diagnostic groups.

One such variable in this context is a policy instrument for the early discharge policy: the average number of patients per bed in a given year for a given hospital. To implement the early discharge policy hospitals may have to increase the number of patients per bed that is allocated to the diagnostic group targeted by the policy. This could be done by either increasing the size of the population served by the hospital through the closure of other hospitals or by reducing the number of beds in the hospital or both. Because some hospitals are likely to attract the severely ill patients the average number of patients per bed in the hospital of delivery could be correlated with the severity of illness. Thus for each observation (patient) I computed the average number patients per bed whether or not it was the choice hospital. The number of patients per bed is a better instrument than the year of policy used in an earlier work [9] because year of policy is only valid as an instrument if no other policy that could affect readmission rate was implemented in the years of and after the policy. Weak instrument test was used to test for the strength of the instrument [10].

The paper is organized as follows. Section 2 describes the model, its estimation and various tests performed. Section 3 describes the data while Section 4 reports the results and Section 5 discusses the results.

\section{METHODS}

\subsection{The Model}

The model for the analysis has two regression equations, an OLS regression Eq.1, and a discrete time duration hazard Eq.2:

$$
\begin{aligned}
& \operatorname{LOS}_{i}=\theta+\delta \mathfrak{J}+\phi Z+u_{i} \\
& y_{i t}=\alpha+\varphi L \hat{O} S+\eta Z+\vartheta_{i t}
\end{aligned}
$$

where $L O S$ represents length of stay in days, $\mathfrak{I}$ is a vector of the instruments, the annual average number of patients per bed for each hospital; $Z$ represents the observable characteristics of the patient: age, income, method of delivery, complications, Indian status, education and birth rate of patient's neighborhood; $u_{i}$ is the unobserved characteristic, severity of illness, of the patient that affects length of stay. Complications is a dummy variable indicating whether the patient's diagnostic short list falls between 147 and 151 which includes hemorrhage of pregnancy, care during pregnancy as well as complications in labor, delivery and pueperium. Note that complication is observable and it differs from severity of illness in that two patients may both have hemorrhage but differ in severity.

In (2), $y_{i t}$ is a binary variable indicating whether or not patient $i$ is readmitted $t$ days ( $t$ is from 1 to ninety) after discharge. Thus $y_{i t}=1$ if the patient is readmitted and zero otherwise. Even though the body takes about 60 days to return to its pre-pregnancy state, when psychological adjustment is taken into account, the woman needs at least 90 days to adjust. Thus 90 days is long enough for any impact of the early discharge policy to be found. The error term $\vartheta_{i t}=v_{i t}+\varepsilon_{i t}$, where $v_{i t}$ is the severity of illness component of $\vartheta_{i t}$ and $\varepsilon_{i t}$ is identically and independently distributed. Thus it is assumed that apart from the severity of illness the error term, $\vartheta_{i t}$, is identically and independently distributed. The presence of severity of illness means that $v_{i t}$ is correlated 
with $u_{i t}$ which implies that LOS is endogenous and the estimates of $\alpha, \varphi$ and $\eta$ are inconsistent.

The hazard equation specifies the probability of being readmitted conditional on not being readmitted. Following the standard data transformation for discrete duration hazard, each patient contributed several observations to the data depending on the number of days, after discharge, it takes before she is readmitted. Thus a patient that is readmitted 18 days after discharge contributes 18 observations to the model. The observations are truncated after 90 days making those not readmitted after 90 days the censored group, each with 90 observations. The transformed data was then estimated by logit.

\subsection{Estimation and Tests}

To technically confirm the endogeneity of length of stay in the readmission equation, the exogeneity test for logit was used to test for the exogeneity of length of stay [11]. The test statistic, $n R^{2}$, was obtained from a regression of a vector of units on $\left[y_{i}-F\left(Z_{i} \hat{\beta}^{M L}\right) X_{i}\right]$ and $\left[y_{i}-F\left(Z_{i} \hat{\beta}^{N L I V}\right) L O S_{i}\right]$ where $\hat{\beta}^{M L}$ is the vector of the estimated parameters in (2) without IV estimation and $\hat{\beta}^{N L I V}$ is the vector of estimated parameters of (2) using IV estimation by nonlinear least squares. Under the null of exogeneity, the test statistic follows $\chi_{G}^{2}$ where $G$ is the number of instrumental variables and $n$ is the sample size.

To ensure the consistent estimation of the coefficients a test for weak instruments as shown in [10] was performed. It is basically a Wald test for the significance of the instruments in (1), i.e., the null hypothesis is $\delta=0$. The test statistic is $\hat{\lambda}^{\prime}\left(\hat{C}^{\prime} \hat{\Omega} \hat{C}\right)^{-1} \hat{\lambda}$, where $\hat{\lambda}$ is a vector of $V$ restrictions, $\delta=0$, evaluated at $\hat{\beta}^{I V}, \hat{C}$ is a $K \mathrm{X} V$ matrix of the derivatives of the restrictions with respect to $\beta^{I V}$, and $\hat{\Omega}$ is the asymptotic Cramer-Rao lower bound variance both evaluated at $\hat{\beta}^{I V}$. The test statistic is $\chi_{V}^{2}$ where $V$ is the number of instruments, which is 16 in this context.

Over identification test [12] was performed to test for the appropriateness of the instruments for length of stay. The null hypothesis states that the instruments are not significant in the readmission equation implying that they are appropriate instruments for length of stay. The test statistic here is also $n R^{2}$ from the least square regression of the residual of (2) on the instruments and the exogenous variables. $n R^{2}$ is also $\chi_{V}^{2}$ where $V$ again is the number of instruments.

\subsection{Data}

The study used a four year (1993-1996) maternity data on all deliveries from sixteen acute care hospitals in
British Columbia, Canada, provided by the British Columbia Ministry of Health and Center for Health Services and Policy Research linked database. For the purposes of this study, the data contained information on age in years, length of stay in days, method of delivery, complications, Indian status, local health area, dates of admission and discharge, transfers and hospital of delivery. Since transfers involve admission and discharges from different hospitals, a transferred patient that returns to her original hospital can easily be mistaken for a readmitted patient. Thus transferred patients were removed from the data. This reduced the sample size from 92,595 to 90,658 deliveries. Readmissions were also reduced from 3492 to 3326. To ensure that readmissions are related to the initial hospitalization only those readmissions caused by obstetric problems were included.

Information on patients' income and educational background were not available and so patients' neighborhood information was used. Neighborhood characteristics of the patients' local health areas for education, income and fertility rates were obtained from the website of the government of British Columbia. The number of maternity beds in each hospital which was needed for the construction of the instruments was obtained from the Directory of Canadian Hospitals. Since the data covered all deliveries in each of the hospitals, the number of patients per bed was computed by dividing the total deliveries in each hospital in a year by the number of maternity beds in the hospital for the year.

As shown in Table 1 the average age of the mothers remained at about 29 over the four years. Length of stay decreased gradually over the years. The number of maternity beds in all the hospitals decreased relative to the number of patients who delivered hence the number of patients per bed increased over time. There was a significant $(17.4 \%)$ reduction in the number of maternity beds in 1994, the year of the policy, followed by smaller $(0.05 \%$ and $3 \%)$ reduction in the two years that followed. The number of maternity beds was therefore reduced over time to implement the early discharge policy hence making it correlate with length of stay.

Table 1. Data summary.

\begin{tabular}{ccccc}
\hline & 1993 & 1994 & 1995 & 1996 \\
\hline Sample size & 23149 & 23325 & 22795 & 22583 \\
$\begin{array}{c}\text { Average } \\
\text { length of } \\
\text { stay }\end{array}$ & 3.71 & 3.47 & 3.29 & 3.22 \\
$\begin{array}{c}\text { Average age } \\
\text { Number of } \\
\text { maternity } \\
\text { beds }\end{array}$ & 29.2 & 29.3 & 29.5 & 29.7 \\
$\begin{array}{c}\text { Patients per } \\
\text { bed }\end{array}$ & 53.6 & 65.3 & 355 & 344 \\
\hline
\end{tabular}


The data used had several advantages in aiding with the consistent estimation of the parameters. First, the omission of breastfeeding and home visit are not likely to affect the consistency of the estimation. In addition to the severity of illness, home visit and breastfeeding are also correlated with both readmission rate and length of stay [8]. As noted in [13], newborns who receive home visits have short stays. Since mothers are unlikely to outstay their babies in the hospital, at least during the period under study, the availability of home care should in general, reduce length of stay for mothers. Home visit also reduces readmissions because the home visit nurse is likely to detect a developing infection and treat before it develops to require readmission. Thus, the omission of home visit can result in inconsistent estimation of the readmission equation. The source of the data used in this study however minimizes any such problem because the early discharge policy in British Columbia was implemented through the introduction of home visit program funded by the Closer to Home Fund available to all hospitals. All patients in the data then had access to home visits and so the impact on readmission is captured by the intercept and not the error term.

Mothers with no lactation problem have short stay and are less likely to be readmitted. Thus the omission of breastfeeding from the estimation equation could render length of stay inconsistent. However, breastfeeding is not likely to affect the consistency of the estimates in the study because lactation problems are captured under complications as care during puerperium. Thus the assumption that without the severity of illness the error term in (2) is identically and independently distributed is valid.

Second, maternity data is appropriate for the study because the early discharge policy has in general being applied to maternity patients across North America and Europe and so the resulting impact on readmission rates of maternity patients have received a lot of attention in the literature $[1,3]$.

Lastly, studies have shown that severity adjusts well when it is disease specific [14]. This is to ensure that the clinical parameters in the model such as complications have similar effect on outcome hence validating the expectation that hemorrhage, for example, increases the probability of readmission rate.

\section{RESULTS}

The test statistic of the endogeneity test was 97835.29 with 16 degrees of freedom and a p-value of zero. Thus the null of no endogeneity was rejected. This confirms the expectation that length of stay is endogenous in the readmission equation; hence previous studies that ignored the endogeneity problem could have produced inconsistent estimates, making their conclusions unreliable.

The test for weak instruments yielded a test statistic of 104458.8 with 16 degrees of freedom and a p-value of zero, leading to the rejection of the null hypothesis of weak instruments. As stated in [10] weak instruments yield inconsistent estimates. The test results then confirm that the instruments used, number of patients per bed, are highly correlated with length of stay and uncorrelated with the severity of illness hence they are not weak instruments for length of stay. Finally the over identification test yielded a test statistic of 14.67 with 16 degrees of freedom and a p-value of 0.547 . Thus, the null was not rejected implying that the instruments are appropriate for length of stay. Having been assured of consistent estimation of the coefficients, attention is now turned to the results of the regression.

As shown in Table 2, all the instrumental variables had negative signs confirming the expectation that length of stay falls as the number of patients per bed increases. The results also showed that length of stay dropped gradually even after 1994, the year of the policy.

The results from the hazard estimation, in Table 2, show that all the estimated coefficients in the IV and the non IV estimation had the same signs. Both show that there was no significant change in readmission rate in, 1995, the year after the policy but readmission rate increased in 1994 and 1996. As expected the readmission rate of those who lived in local health areas with high education level and/or high income were less likely to be readmitted conditional on not yet been readmitted. Patients with complications as well as those with Native Indian status were more likely to be readmitted conditional on not yet been readmitted, than those without complications and/or Native Indian status. As expected, Native Indians were more likely to be admitted than non-native Indians. In general, the IV estimates were less efficient than the non-IV estimates.

Since readmission rates are considered often as unconditional, unconditional readmission rates were computed to compare the impact of the policy on readmission rate using the IV and the non-IV estimations. The computation involves calculating, for each patient, the survival rate, subtracting it from one and then averaging over all the patients. The coefficient for length of stay under the non-IV was $-0.051+0.0008^{*} L O S$ and that of the IV was $-0.115+0.006 * L O S$. The average length of stay of 1994 in the above coefficients and unconditional readmission rates were used to compute the marginal effect of length of stay on readmission rate. The results showed a marginal effect of $-1.54 \%$ under the IV estimation and $-1.1 \%$ under the non-IV estimation, i.e., an increase in length of stay by a day reduced readmission rate by $1.54 \%$ under the IV estimation and $1.1 \%$ under 
Table 2. Regression results (numbers are estimated coefficients).

\begin{tabular}{|c|c|c|c|}
\hline $\begin{array}{ll}\text { Independent } & \text { Dependent Variables } \\
\text { Variables } & \end{array}$ & Length of Stay & $\begin{array}{l}\text { Readmission Equation } \\
\text { (with IV estimation) }\end{array}$ & $\begin{array}{l}\text { Readmission Equation } \\
\text { (without IV estimation) }\end{array}$ \\
\hline Number of patients per bed 1 & -0.00003 & & \\
\hline Number of patients per bed 2 & 0.0002 & & \\
\hline Number of patients per bed 3 & -0.015 & & \\
\hline Number of patients per bed 4 & -0.007 & & \\
\hline Number of patients per bed 5 & -0.028 & & \\
\hline Number of patients per bed 6 & -0.016 & & \\
\hline Number of patients per bed 7 & -0.019 & & \\
\hline Number of patients per bed 8 & -0.032 & & \\
\hline Number of patients per bed 9 & -0.043 & & \\
\hline Number of patients per bed 10 & -0.167 & & \\
\hline Number of patients per bed 11 & -0.026 & & \\
\hline Number of patients per bed 12 & -0.150 & & \\
\hline Number of patients per bed 13 & -0.00005 & & \\
\hline Number of patients per bed 14 & -0.091 & & \\
\hline Number of patients per bed 15 & -0.015 & & \\
\hline Number of patients per bed 16 & -0.047 & & \\
\hline 1994 & -0.174 & 0.104 & 0.105 \\
\hline 1995 & -0.344 & 0.002 & 0.008 \\
\hline 1996 & -0.404 & 0.092 & 0.103 \\
\hline Income & 0.841 & -0.174 & -0.175 \\
\hline Native Indians & 0.130 & 0.277 & 0.283 \\
\hline Fertility & 0.239 & 0.097 & 0.103 \\
\hline Age & -0.122 & -0.073 & -0.092 \\
\hline Age*age & 0.002 & 0.001 & 0.002 \\
\hline Education & -0.897 & -0.142 & -0.154 \\
\hline Complication & 0.450 & 0.544 & 0.523 \\
\hline Length of stay & & -0.115 & -0.051 \\
\hline Length of stay * length of stay & & 0.031 & 0.0001 \\
\hline $\mathrm{T}$ & & -0.59 & -0.059 \\
\hline $\mathrm{T} 2$ & & 0.001 & 0.001 \\
\hline constant & 5.288 & -5.477 & -5.431 \\
\hline
\end{tabular}

All estimates are significant at $5 \%$ significance level.

the non-IV estimation. Thus, there is a greater marginal impact of length of stay on readmission rate under the IV than the non IV estimation. This result is similar to that in [8] as well as in [6]. 
An LM test was run to check if the coefficients of length of stay in the IV estimation were statistically different from those of non-IV. This null hypothesis is $\varphi^{I V}=$ $\varphi^{n o n I V}$. The test involves running the residuals of the restricted regression on the derivatives of the logit with respect to each of the coefficients in the model. The test statistic is $n R^{2}$ which follows the chi square distribution with two degrees of freedom (number of restrictions). The resulting test statistic was 2445.7 with a p-value of zero, implying that the coefficients were statistically different from each other. Hence the earlier studies that ignored the endogeneity of length of stay could be flawed.

The extend to which the policy contributed to the increase in readmission rates was found by rerunning the readmission regression after including interactions of length of stay with the year dummies. The resulting estimated coefficients for the interactions of the year dummies with the square of length of stay were not significant for either the IV or the non-IV estimates so the regressions were reran without those interactions. The results are reported in Table 3 and they show that the interaction coefficients for the years and length of stay were negative for the IV results and positive but close to zero for the non-IV results. This implies that according to the IV results, the reduction in readmission rate from a day's increase in length of stay is greater after the period of the policy. The opposite is the case under the non-IV results.

To translate these into readmission rates, the results in Table 3 were used to compute the contribution of the policy to any change in readmission rate as the difference between the readmission rates with and without the interactions. The readmission rates without the interaction were the readmission rates for each year without any contribution from the early discharge policy. The readmission rates that included the interactions represented the readmission rates for each year including the impact of the policy. The difference between the two then is the change in readmission rate as a result of the policy. To compare the impact of the policy with the overall change in readmission rate over time, the results in Table 2 were used to compute the change in readmission rate for each year after the policy.

The results, reported in Table 4, show that readmission rates increased over the years under both the IV and the non-IV estimation. However, the increase was consistently higher under the IV than the non-IV estimation. The results under IV also showed that with the exception of 1994, where other factors must have contributed to the increase in readmission rate, any increase in readmission rate in the years that followed was due to the policy and other factors must have reduced the impact of the policy. Such information was not captured by the non-IV results.
Table 3. Regression results from interacting length of stay with the year dummy variables.

\begin{tabular}{|c|c|c|}
\hline & $\begin{array}{l}\text { Readmission Rate } \\
\text { (with IV) }\end{array}$ & $\begin{array}{l}\text { Readmission rate } \\
\text { (without IV) }\end{array}$ \\
\hline $1994^{*}$ & 0.128 & 0.095 \\
\hline $1995^{*}$ & 0.111 & -0.023 \\
\hline $1996^{*}$ & -0.212 & 0.050 \\
\hline $1994 *$ length of stay ${ }^{*}$ & -0.004 & 0.002 \\
\hline $1995^{*}$ length of stay ${ }^{*}$ & -0.027 & 0.007 \\
\hline $1996 *$ length of stay ${ }^{*}$ & 0.102 & 0.013 \\
\hline Income & -0.174 & -0.177 \\
\hline Native Indians & 0.275 & 0.283 \\
\hline Fertility & 0.100 & 0.103 \\
\hline Age & -0.079 & -0.092 \\
\hline Age*age & 0.001 & 0.002 \\
\hline Education & -0.141 & -0.155 \\
\hline Complication & 0.494 & 0.523 \\
\hline Length of stay ${ }^{*}$ & -0.036 & 0.045 \\
\hline $\begin{array}{l}\text { Length of stay* } \\
\text { length of stay }\end{array}$ & 0.017 & -0.0004 \\
\hline $\mathrm{T}$ & -0.059 & -0.059 \\
\hline $\mathrm{T} 2$ & 0.001 & 0.001 \\
\hline constant & -5.516 & -5.406 \\
\hline
\end{tabular}

"Significant at $10 \%$ level. All other variables are significant at $5 \%$ significance level.

Table 4. Effect of policy on readmission rates.

\begin{tabular}{lcccccc}
\hline & \multicolumn{2}{c}{1994} & \multicolumn{2}{c}{1995} & \multicolumn{2}{c}{1996} \\
\hline & IV & Non-IV & IV & Non-IV & IV & Non-IV \\
\hline $\begin{array}{l}\text { Change in } \\
\text { readmission } \\
\text { rate (\%) }\end{array}$ & 2.8 & 2.6 & 2.1 & 0.19 & 2.6 & 2.5 \\
$\begin{array}{l}\text { Change in } \\
\text { readmission } \\
\text { rate due to } \\
\text { policy (\%) }\end{array}$ & 1.5 & 1.1 & 2.4 & 0.23 & 5.2 & .101 \\
$\begin{array}{l}\text { Number of } \\
\text { readmissions } \\
\text { due to policy }\end{array}$ & 536 & 23 & 547 & 52 & 1174 & 23 \\
\hline
\end{tabular}

As shown in Table 4, for 1994 the policy increased readmission rate by 0.1 percentage points under the non-IV estimation and 2.3 percentage points under the IV estimation. Considering the number of deliveries of 23,325 in the selected hospitals in 1994, it implies, according to the non-IV estimation about only 23 women were readmitted as a result of the early discharge policy. 
The number however increased to about 536 women under the IV estimation.

\section{CONCLUSIONS}

The current study has one main weakness. The IV estimation, when the second stage regression is logit or probit, requires that the endogenous variable is continuous. This implies that length of stay should be measured in hours and not in days as was used. The requirement that the problem variable be continuous is consistent with probit estimation which is continuous. However, in the current study the second stage regression is discrete and so a discrete endogenous variable for the first stage may not be problematic.

The study however has several strengths. First, the instruments, average patients per bed, proposed to ensure consistent estimation of the impact of the early discharge policy on readmission rate are not weak. Second the instruments are not significant in the readmission equation implying that they are not one of the regressors for readmission. Hence they are strong and appropriate instruments. Third, the instruments are not restricted to any diagnostic group and so can widely be applied to any diagnostic group to find consistent estimation of the impact of the early discharge policy. Finally, information on the number of beds for a diagnostic group can easily be found for the computation of the average number of patients per bed.

The study has shown that the non-IV estimates are statistically different from the IV estimates implying that earlier studies that ignored the endogeneity of length of stay might have produced misleading results. The nonIV estimates underestimate the impact of the policy on readmission rate. That could explain why some of the studies that ignored the endogeneity problem found no impact of the policy on readmission rate. The results from the non-IV estimates inform policy makers that the policy as it was implemented did not deteriorate readmission rate and so provided no reason for policy makers to make any adjustment. The results from the IV estimates imply that, since readmissions are expensive, the policy as implement might not contain as much cost as was expected and so further amendments such as improvement in home care is necessary to make the policy more able to reduce the cost of care.

\section{REFERENCES}

[1] Thomspon, A.H., Sauders, A.L.D., Cumming D.C. and Thanigasalam, N. (2003) Post-maternity outcomes following health care reform in Alberta: 1992-1996. Canadian Journal of Public Health, 94(4), 104-108.

[2] Gazmararian, J.A. and Koplan, J.P. (1996) Length-ofstay after delivery: Managed care versus fee-for-service.
Health Affairs, 15(4), 74-80.

[3] Kosecoff, J., Kahn, K., Rogers, W., Reinisch, E., Sherwood, M., Rubenstein, L., Draper, D., Roth, C., Chew, C. and Brook, R. (1990) Prospective payment system and impairment at discharge: 'The quicker and sicker' story revisited. Journal of the American Medical Association, 264(15), 1980-1983.

[4] Tai-Seale, M., LoSasso, A.T., Freund, D.A. and Gerber, S.E. (2001) The long-term effects of medicaid managed care on obstetric care in three California counties. Health Services Research, 36(4), 751-771.

[5] Rubenstein, L., Kahn, K., Reinisch, E., Sherwood, M., Rogers, W., Karnberg, Draper, D. and Brook, R. (1990) Changes in quality of care for five diseases measured by implicit review. Journal of the American Medical Association, 264(15), 1981-1986.

[6] Kahn, K., Rogers, W., Rubenstein, L., Sherwood, M., Reinisch, E., Keeler, E., Draper, D., Kosecoff, J. and Brook. R. (1990) Comparing outcomes of care before and after implementation of the DRG-based prospective payment system. Journal of the American Medical Association, 264(15), 1984-1988.

[7] Keeler, E., Kahn, K., Draper, D., Sherwood, M., Rubenstein, L., Reinisch, E., Kosecoff, J. and Brook, R. (1990) Changes in sickness at admission following the introduction of the prospective payment system. Journal of the American Medical Association, 264, 1962-1968.

[8] Iezzoni, L.I., (1994) Risk adjustment for measuring health care outcomes. Health Administration Press, Ann Arbor, IM.

[9] Gowrinsandaran, G. and Town, R.J. (1999) Estimating the quality of care in hospitals using instrumental variables. Journal of Health Economics, 18(6), 747-767.

[10] Heggestad, T. (2002) Do hospital length of stay and staffing ratio affect elderly patient's risk of readmission? A nation-wide study of Norwegian hospitals. Health Services Research, 37(3), 647-665.

[11] Malkin, J.D., Broder, M.S. and Keeler, E. (2000) Do longer postpartum stays reduce newborn readmissions' analysis using instrumental variables. Health Services Research, 35(5), 1071-1091.

[12] Amporfu, E. (2008) Quality effect of early discharge of maternity patients: Does hospital specialization matter? Forum for Health Economics \& Policy. Health Econmics, 11(2). http://www.bepress.om/fhep/11/2/11

[13] Staiger, D. and Stock, J.H. (1997) Instrumental variables regression. Econometrica, 65, 557-586.

[14] Grogger, J. (1990) A simple test for exogeneity in probit and logit, and poisson regression models. Economics Letters, 33(4), 329-332.

[15] Hensen, L. (1982) Large sample properties of generalized method of moments estimators. Econometrica, 50(4), 1029-1054.

[16] Gazmararian, J.A., Koplan, J.P., Cogswell, M.E., Bailey, C.M., Davis, N.A. and Cutler, C.M. (1997) Maternity experiences in a managed care organization. Health Affairs, 16(3), 198-208

[17] Wray, N.P., Hollingsworth, J.C., Petersen, N.J. and Aston, C.M. (1997) Case-mix adjustment using administrative databases: A paradigm to guide future research, Medical Care Research and Review, 54(3), 326-356. 